\title{
La contención emocional. Un camino para conservar la estabilidad emocional en el personal que atiende a mujeres víctimas de violencia.
}

The emotional restraint. A way to maintain emotional stability in the caring staff of women victims of violence

Irma Carrillo Flores

Universidad Autónoma de Aguascalientes

irmairma9@hotmail.com

\section{Resumen}

En el marco del proyecto de investigación: aplicación a nivel piloto de los modelos de prevención, atención, sanción y erradicación de la violencia de género contra las mujeres, desarrollado en la Universidad Autónoma de Aguascalientes durante 2011, después de capacitar a 139 personas de las dependencias: CAPIV, Instituto Aguascalentense de las Mujeres, Instancias Municipales, Maestros universitarios y Servidores públicos del Gobierno del Estado de Aguascalientes se hizo visible la necesidad de cuidado emocional del personal que atiende a mujeres víctimas de violencia para lograr un bienestar laboral y tener una mejor calidad de vida.

Las personas capacitadas reconocieron, cuando se trabajó con ellas en grupos de encuentro, que deben conocerse a sí mismos para no repetir en forma automática e inconsciente pautas de conducta conocidas cuando se está en contacto con situaciones de violencia que de alguna u otra manera remiten a vivencias similares. Reconocieron que el ejercicio de Contención entre pares y el realizado por expertos permite que estos profesionistas que trabajan con mujeres que 
han sido violentadas no comprometan su ejercicio profesional con situaciones personales o asuntos inconclusos personales.

Palabras clave: Bienestar laboral, grupos de encuentro, género, calidad de vida, contención emocional.

\section{Abstract}

As part of the research project: application to pilot models prevention, treatment, punishment and eradication of violence against women, developed at the Autonomous University of Aguascalientes in 2011 level after train 139 people from the dependencies: CAPIV, Aguascalentense women's Institute, Municipal Instances, university teachers and public servants of the Government of the State of Aguascalientes was visible the need for emotional care workers who treat women victims of violence to achieve a workplace wellness and a better quality of life.

Key words: emotional support, stability, violence, women.

Fecha recepción: Octubre $2011 \quad$ Fecha aceptación: Noviembre 2011

\section{Introducción.}

El propósito de este texto es mostrar los resultados de la implementación de grupos de encuentro con 139 personas que trabajan con mujeres que son violentadas en el Estado de Aguascalientes Se realizaron talleres para la trasmisión de conocimientos y apropiación de los Protocolos de Actuación en Aguascalientes a servidoras y servidores públicos relacionados con las políticas públicas sobre violencia de género contra las mujeres. 
Para obtener información de quienes participaron en las sesiones de capacitación se realizaron consultas utilizando los modelos y flujo-gramas existentes, sobre los Protocolos de Actuación. Se realizaron reuniones con el total de personas que asistieron a las capacitaciones, para discutir sobre el contenido de las sesiones de capacitación recién realizadas. Los temas fueron los Protocolos de Prevención, Atención, Contención, Acompañamiento y Trabajo con varones agresores. Cada uno de los grupos de discusión se llevo a cabo con los temas tratados en la capacitación.

En este documento se describe el instrumento con el que se recabó la información que aquí se reporta, el objetivo de este instrumento, los sujetos de los que se obtuvo la información, los resultados generales sobre la información vertida en los grupos de discusión. Finalmente, se presentan recomendaciones para el desarrollo de futuras capacitaciones.

En suma, en este trabajo que hace evidente la necesidad de que el personal que se enfrenta con las situaciones y circunstancias que la mujer víctima de violencia le presenta, se atienda de manera pertinente y se beneficie de un programa de contención para lograr el restablecimiento del equilibrio emocional y su desempeño óptimo.

\section{Desarrollo de contenidos sobre la temática seleccionada.}

Los grupos de discusión son un instrumento del campo de las Ciencias Sociales que permitió en esta investigación, obtener información sobre las opiniones de los participantes de las capacitaciones. El objetivo de usar este instrumento fue adquirir más información provocando auto confesiones en las y los participantes, logrando una retroalimentación sobre el protocolo de contención revisado en la capacitación.

El número de entrevistados fue el total de los participantes en las capacitaciones, misma(o) $\mathrm{s}$ que por sexo se distribuyen como se muestra en el cuadro 1

\section{Cuadro 1.}

Participantes en la consulta de los protocolos de actuación por sexo 


\begin{tabular}{|l|l|l|l|l|l|}
\hline Grupo/Aspecto & hombres & $\%$ & mujeres & $\%$ & TOTAL \\
\hline Grupo A & 16 & 24.24 & 50 & 75.75 & 66 \\
\hline Grupo B & 4 & 21.05 & 15 & 78.94 & 19 \\
\hline Grupo C & 13 & 24.07 & 41 & 75.92 & 54 \\
\hline Totales & 33 & & 106 & & 139 \\
\hline
\end{tabular}

En el cuadro 1 es notorio que son más las mujeres que participan en las Instituciones que tienen alguna relación con mujeres que han sido violentadas. El total de participantes según el grupo en el que fueron capacitados se distribuye como lo muestra el cuadro 2

\section{Cuadro 2}

Participantes de la capacitación por grupo

\begin{tabular}{|l|l|}
\hline Grupo A & $\begin{array}{l}\text { Personal del Instituto Aguascalentense de las Mujeres (IAM) } \\
\text { Personal del Centro de Atención y Prevención Integral de la } \\
\text { Violencia (CAPIV) y representantes de municipios. } \\
\text { Personal de CAPIV y representantes de municipios (segunda } \\
\text { capacitación). }\end{array}$ \\
\hline Grupo B & Personal de la Universidad Autónoma de Aguascalientes (UAA). \\
\hline Grupo C & $\begin{array}{l}\text { Personal de las Instituciones Integrantes del Consejo para erradicar } \\
\text { la violencia de género. } \\
\text { Primera reunión de trabajo con personal de: } \\
\text { Secretaría de seguridad Publica } \\
\text { Procuraduría General de Justicia } \\
\text { Instituto de Salud del Estado de Aguascalientes } \\
\text { Fundación Mujer Contemporánea }\end{array}$ \\
\hline
\end{tabular}




\begin{tabular}{|l|l|}
\hline $\begin{array}{l}\text { Colectivo Hombres Libres de Violencia. } \\
\text { Personal de Desarrollo Integral de la Familia (DIF) - Estatal }\end{array}$ \\
\hline
\end{tabular}

Fuente. Elaborado por Irma Carrillo Flores, mayo 2011, UAA. En el desarrollo de la investigación: Aplicación a nivel Piloto de los modelos de PreVención, Atención, Sanción Y ERRADICACIÓN DE LA VIOLENCIA dE GÉNERO CONTRA LAS MUJERES EN EL ESTADO dE AgUASCALIENTES.

Para el desarrollo de la Consulta fue necesario contar con una facilitadora o facilitador que dirigiera el desarrollo de la reunión y una relatora o relator, que tomara nota de todo lo que se diga en la misma. Las funciones de la facilitadora son: iniciar la reunión, presentar el tema, asegurarse de que todos puedan emitir su opinión, organizar las exposiciones, evitando las participaciones superpuestas, mediar o evitar conflicto entre quienes participan, promover acuerdos, de ser necesario y cerrar la reunión.

Por su parte, las funciones de la relatora o relator son: tomar nota de todo lo dicho por quienes participan en la reunión. Por escrito, haciendo uso de la tecnología, mediante grabación de audio o de video, dar lectura a información que genere la participación de quienes estén presentes. Si el caso lo amerita, elaborar una síntesis de lo expuesto, las conclusiones obtenidas, incluyendo los disensos y leer la información registrada, a modo de resumen de la reunión.

Los participantes, incluidos facilitadora o facilitador y relatora o relator, son responsables de: aportar sus conocimientos y opiniones sobre el tema en forma simple y precisa, hablar con voz clara y audible, ser moderados y respetuosos en el uso del tiempo disponible, respetar opiniones disidentes y estar atentos para no repetir ideas.

Participantes en la consulta.

Los 139 participantes en los grupos de discusión en promedio fueron $75 \%$ mujeres y $25 \%$ hombres. Atendiendo a sus edades, en promedio predominaron los adultos jóvenes. En cuanto a la Responsabilidad en la Administración Pública, la mayoría son mandos medios y solo la minoría funcionarios de alto nivel. Para realizar la capacitación se tomó en cuenta primeramente el conjunto de dependencias que la Ley de acceso a las mujeres a una vida libre 
de violencia del Estado de Aguascalientes establece como responsables de implementar acciones tendientes a la erradicación de la violencia de género contra las mujeres.

Instrumento de recolección de información

El instrumento que usa la relatora para trabajar en las sesiones y garantizar la obtención de información, durante el desarrollo de los grupos de discusión es una guía de entrevista abierta colectiva que se compone del Modelo gráfico del protocolo de Acompañamiento y los flujogramas de los protocolos de Atención, Contención y Tratamiento de agresores. Este documento es el apoyo de la coordinadora. Le sirve para poder guiar a los participantes y respetar los lineamientos que se requieren, así como obtener la información de acuerdo a los temas a considerar.

La elección de los factores o variables a indagar en el grupo de discusión se realizó en función de los objetivos del proyecto de investigación que son: realizar talleres para la trasmisión de conocimientos y apropiación de los protocolos de actuación en Aguascalientes a servidores públicos relacionados los siguientes temas: Viabilidad de los protocolos; Fortalezas; Áreas con las políticas públicas de violencia contra las mujeres y realizar informe de resultados de la aplicación de los protocolos para el análisis del desarrollo del proyecto de investigación.

Primero que nada se entrega a los participantes el modelo gráfico y los flujo-gramas correspondientes a cada protocolo, como recordatorio de lo visto en la capacitación y para facilitar su participación en el grupo de discusión. Este elemento lo llamaremos: Conocimiento de los protocolos de actuación.

El siguiente elemento a indagar fue la viabilidad de los protocolos, posteriormente se recupera información sobre fortalezas, debilidades y sugerencias para mejorar estos protocolos. La información obtenida, posteriormente fue la materia prima para realizar mapas conceptuales con ayuda de la herramienta computarizada del mindjet Mind Manager.

Herramienta para la sistematización de información: El Mindjet MindManager 
Gran parte del esfuerzo profesional y organizacional de hoy es improductivo porque las metodologías y herramientas tradicionales son insuficientes para el mundo de hoy. El MindJet MindManager ayuda a las personas y organizaciones a lograr resultados sobresalientes al ofrecer metodologías y consultoría que junto con tecnologías de punta desarrollan la inteligencia colectiva y la innovación de su organización. La información es poder cuando se tiene la capacidad de procesarla y convertirla en acciones. Mindmanager acelera y multiplica la gestión integral de información.

En suma, el MindJet MindManager es una herramienta práctica y efectiva en la gestión profesional para visualizar información. En el contexto de la presente investigación, las opiniones de los participantes se fueron vaciando una a una en un mapa conceptual hasta que la idea se hizo repetitiva. Se concentró la información protocolo por protocolo hasta armar un mapa conceptual por grupo capacitado.

Técnica de análisis de información:

Como se señaló en el apartado anterior, para analizar la información se utilizaron mapas conceptuales $^{1}$ generados en el programa MindJet MindManager, que posteriormente son trabajados a manera de matrices en las que se enlistan las aportaciones más significativas de quienes participaron, así como las áreas de oportunidad de las que se habló en las sesiones.

Los mapas conceptuales son instrumentos de representación de los conocimientos sencillos y prácticos, que permiten transmitir con claridad mensajes conceptuales complejos y facilitar tanto el aprendizaje como la enseñanza. Para mayor abundamiento, adoptan la forma de grafos.

\footnotetext{
${ }^{1}$ Representación visual bidimensional de los conceptos y la relación entre conceptos en relación con un tema, organizado jerárquicamente de lo general a lo particular.
} 
Los mapas conceptuales son artefactos para la organización y representación del conocimiento. Tienen su origen en las teorías sobre la psicología del aprendizaje de David Ausubel enunciadas en los años 60, su objetivo es representar relaciones entre conceptos en forma de proposiciones. Los conceptos están incluidos en cajas o círculos, mientras que las relaciones entre ellos se explicitan mediante líneas que unen sus cajas respectivas. Las líneas, a su vez, tienen palabras asociadas que describen cuál es la naturaleza de la relación que liga los conceptos $^{2}$. Una proposición es una frase acerca de cierto objeto o suceso en el universo, que ocurre de forma natural o artificial. Las proposiciones contienen dos o más conceptos conectados con otras palabras que forman una frase coherente. Se las suele llamar "unidades semánticas". Un mapa conceptual se forma por conceptos enlazados por relaciones, cajas y líneas que las unen, los mapas conceptuales se pueden representar y de hecho se representan, mediante grafos en los que los nodos son los conceptos y los arcos las relaciones entre ellos. Los mapas conceptuales se estructuran en forma jerárquica en la que los conceptos más generales están en la raíz del árbol y a medida que vamos descendiendo por el mismo nos vamos encontrando con conceptos más específicos ${ }^{3}$.

Resultados de los grupos de discusión.

En este apartado se presentan varias matrices, que sintetizan las opiniones de los participantes en la consulta de los protocolos de actuación por grupo. Se destaca la información obtenida en dos rubros: fortalezas observadas en el protocolo de contención para su implementación y las áreas de oportunidad en las que es necesario trabajar con el mismo en el Estado de Aguascalientes.

\footnotetext{
2 Joseph D. Novak en el artículo "The Theory Underlying Concept Maps and How To Construct Them" define concepto como "una regularidad percibida en sucesos u objetos o registros de sucesos u objetos, designado por una etiqueta". La etiqueta de un concepto es usualmente una palabra.

3 (http://www.infovis.net/printMag.php?num=141\&lang=1. Artículo escrito por Juan C. Dürsteler, consultado el 03/06/2011).
} 
Con el fin de organizar la información de manera que los resultados sean representativos y evitar sesgos, se integró a los participantes en grupos. Estos grupos de informantes fueron tres, según sus características o procedencia institucional. Serán denominados en lo sucesivo: Grupo A, Grupo B y Grupo C.

Se realizaron nueve grupos de discusión en el transcurso de seis sesiones de capacitación. En la primer sesión se llevaron a cabo tres grupos de discusión; en la segunda sesión se realizó un grupo de discusión; en la tercera sesión uno grupo de discusión; en la cuarta uno ${ }^{4}$, en la quinta uno y en la sexta dos.

Sistematizada la información que se obtuvo en el ejercicio de los Grupos de Discusión, se observan más similitudes que diferencias en los resultados de los grupos. Sin embargo, se presentan estos por grupo de procedencia institucional, con el fin de obtener las visiones particulares de cada uno de ellos y estar en posibilidades de construir una visión de conjunto.

Resultados por grupo institucional

\section{Matriz 1}

Resultados del Grupo A

\begin{tabular}{|l|l|l|}
\hline GRUPO & FORTALEZAS & ÁREAS DE OPORTUNIDAD \\
\hline & Protocolo de contención: & Protocolo de contención \\
\hline & & \\
& La mayor fortaleza es la & Se tiene falta de experiencia en la \\
& existencia del protocolo de & mayoría de los servidores públicos en \\
\hline
\end{tabular}

\footnotetext{
4 La información obtenida en esta sesión a la que acudieron las Asociaciones Civiles se procesó de manera conjunta con el grupo C donde se capacitó al personal de las Instituciones Integrantes del Consejo de Equidad de Género, Secretaría de seguridad Publica Procuraduría General de Justicia, Instituto de Salud del Estado de Aguascalientes y Personal del DIF - Estatal.
} 


$\begin{array}{lll}\text { Personal } & \text { contención mismo y la el tema, ya que tienen poco tiempo en } \\ \text { CAPIV } & \text { existencia de las Instancias } & \text { el cargo y este taller y las experiencias } \\ \text { IAM } & \text { municipales y la voluntad } & \text { compartidas en el grupo son su primer } \\ \text { Instancias } & \text { política para aplicarlo, de parte } & \text { acercamiento al mismo. } \\ \text { municipales } & \text { del Instituto Aguascalentense de }\end{array}$

Fuente. Elaborado por Irma Carrillo Flores y Montserrat Lara Zapata, mayo 2011, UAA.

En el desarrollo de la investigación: ApLICACIÓn A NIVEL PILOTO DE LOS MOdelos DE PREVENCIÓN, ATENCIÓN, SANCIÓN Y ERRADICACIÓN DE LA VIOLENCIA DE GÉNERO CONTRA LAS MUJERES EN EL ESTADO DE Aguascalientes.

La principal fortaleza del protocolo de contención es el protocolo mismo pero la principal área de oportunidad es la cantidad insuficiente de personal destinado al área de atención psicológica que pueda atender a las mujeres que sufren violencia y al personal que trabaja con estas mujeres.

Con el grupo B, Profesores Universitarios, no se trabajó con el protocolo de contención por lo tanto no se hizo grupo de discusión sobre ese protocolo. En el grupo C los resultados fueron:

Matriz 3

Resultados del grupo C

\begin{tabular}{l|l|l}
\hline GRUPO & FORTALEZAS & ÁREAS DE OPORTUNIDAD
\end{tabular}




\begin{tabular}{|c|c|c|}
\hline $\begin{array}{l}\text { Integrantes } \\
\text { del } \\
\text { Consejo } \\
\text { para } \\
\text { erradicar la } \\
\text { violencia } \\
\text { de género }\end{array}$ & $\begin{array}{l}\text { Protocolo de Contención: } \\
\text { Contar con el Protocolo ya que es } \\
\text { muy completo, específico y muy } \\
\text { importante, porque ya no solo se } \\
\text { está pensando en la mujer que sufre } \\
\text { violencia sino también en la persona } \\
\text { que la atiende. }\end{array}$ & $\begin{array}{l}\text { Protocolo de Contención: } \\
\text { Falta de recurso económico para que } \\
\text { se brinde Contención a las y los } \\
\text { servidores públicos. La falta de } \\
\text { contención genera estrés en quienes } \\
\text { atienden a las mujeres violentadas de } \\
\text { forma directa y provoca atención de } \\
\text { mala calidad. }\end{array}$ \\
\hline
\end{tabular}

Fuente. Elaborado por Irma Carrillo Flores, y Montserrat Lara Zapata, mayo 2011, UAA.

En el desarrollo de la investigación: ApLICACIÓn a NIVEL PILOTO de los MOdelos de PREVEnCión, ATENCIÓN, SANCIÓN Y ERRADICACIÓN DE LA VIOLENCIA DE GÉNERO CONTRA LAS MUJERES EN EL ESTADO DE Aguascalientes.

Al igual que en el grupo A, la principal fortaleza del protocolo de contención es el protocolo mismo y la principal área de oportunidad es la cantidad de personal destinado al área de atención psicológica que pueda atender a las mujeres que sufren violencia y al personal que trabaja con estas mujeres. A continuación se muestra, a manera de ejemplo, el mapa conceptual de las opiniones vertidas en el grupo A

Consulta al Grupo A

Mapa conceptual 1 
Personal del IAM, CAPIV e Instancias Municipales

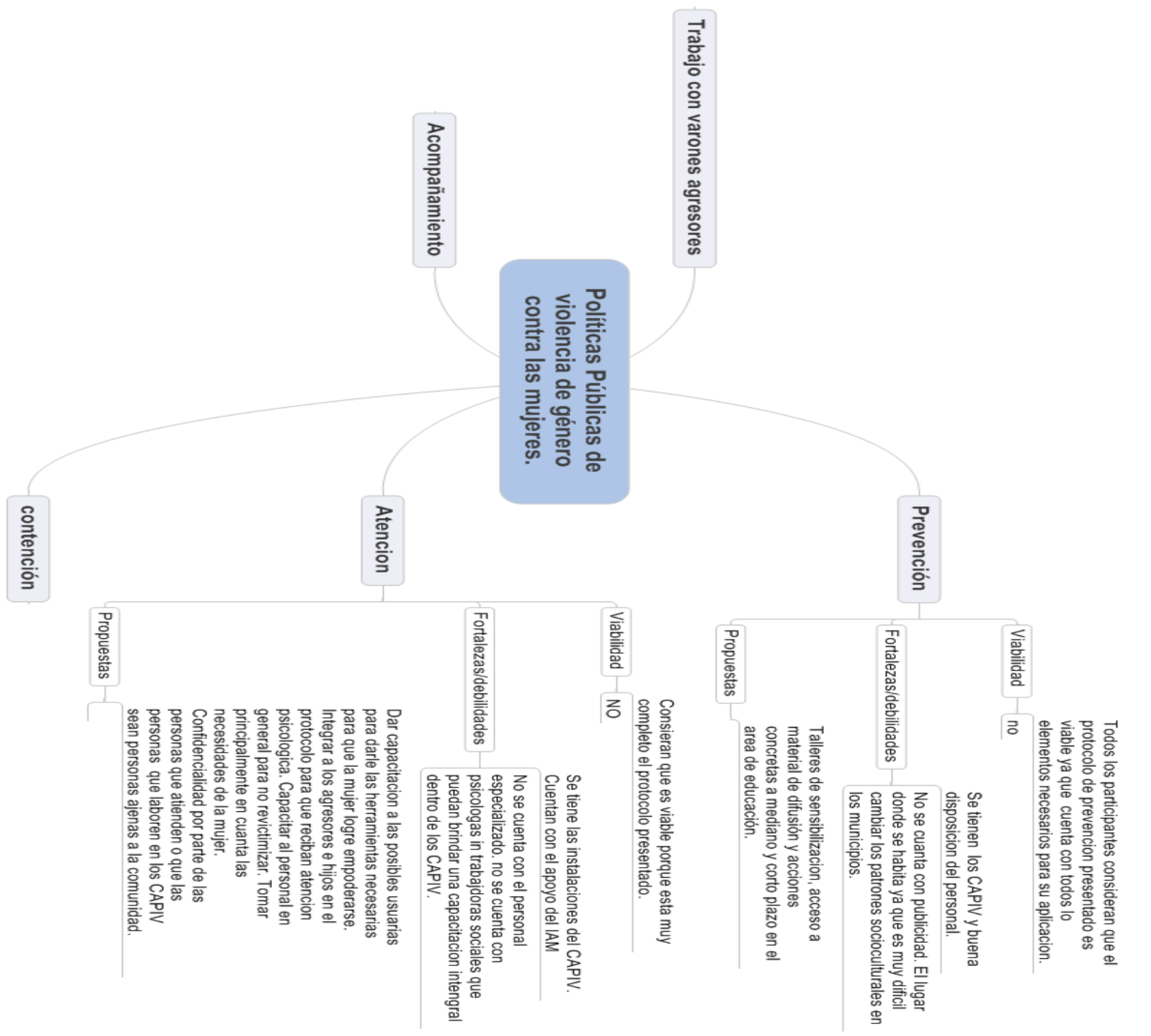

Respecto al protocolo de contención, éste no solo lo consideran viable sino necesario para las y los funcionarios que atienden violencia de género en contra de las mujeres.

Para los participantes la mayor fortaleza es la existencia del protocolo de contención mismo y la existencia de las Instancias municipales y el Instituto Aguascalentense de las Mujeres. El área 
de oportunidad que se tiene es la falta de experiencia ya que la mayoría de los servidores públicos tienen poco tiempo en el cargo y este taller y las experiencias compartidas en el grupo son su primer acercamiento en el tema.

La propuesta que los participantes hacen para poder implementar el protocolo de contención de una manera eficiente es la coordinación por parte de los CAPIV y las Instancias Municipales para que cuenten con las herramientas necesarias para brindar contención a los municipios y a todo el personal especializado en brindar atención a mujeres que sufren violencia.

Respecto al protocolo de contención, todos los grupos consideran que es viable y necesario para las y los servidores públicos. Consideran que la mayor fortaleza es ahora contar con el protocolo ya que es muy completo y especifico y muy importante porque ya no solo se está pensando en la mujer que sufre violencia sino también en la persona que la atiende.

Una de las áreas de oportunidad de este protocolo que expresan las y los participantes es la falta de recurso económico para que se brinde contención a las y los servidores públicos es la mayor área de oportunidad y como consecuencia se genera mucho estrés y provoca atención de mala calidad.

\section{Conclusiones}

Las propuestas que hicieron los participantes en los grupos de discusión se sintetizan de la siguiente manera: 1) que se le de mayor difusión al protocolo de contención entre las diferentes instancias que se encargan de atender a mujeres que sufren violencia. 2) Se propone que para llevar a cabo la contención se requiere de psicólogos externos a las instituciones que atienden mujeres que han sido violentadas, para sentir mayor confianza al compartir cuestiones personales y laborales. 3) Sensibilizar a los directivos para que estén conscientes de que los profesionales que atienden a mujeres que sufren violencia necesitan desintoxicarse 4) Que para poder implementar el protocolo de contención de una manera eficiente se debe dar una coordinación adecuada por parte de los CAPIV y las Instancias Municipales para que cuenten con las herramientas necesarias para brindar contención al personal que trabaja en los 
municipios y a todo el personal especializado en brindar atención a mujeres que sufren violencia.

\section{Bibliografia}

ÁlVAREZ de Lara, R. M. Pérez, A. E. \& Noroña (2010). Modelos para prevenir, atender, sancionar y erradicar la violencia contra las mujeres. Universidad Nacional Autónoma de México y Consejo Nacional de Ciencia y Tecnología.

IBÁÑEZ, Jesús (1979). El grupo de discusión, teoría y crítica. México: Siglo XXI Editores.

INMUJERES (2006): PROEQUIDAD. Programa Nacional de Igualdad de Oportunidades y no Discriminación contra las Mujeres. México.

RODRÍGUEZ Gallardo, Georgina Ligeia (2011). Violencia en el noviazgo, el juego de la violencia con cariño. Tesis de doctorado de la Universidad Autónoma de Aguascalientes, Aguascalientes, México. 